Voix et Images

volxetimages

\title{
Le théorique, la politique, Bakhtine et la sociocritique
}

Relire Belleau

\section{Theory, Politics, Bakhtin and Sociocriticism}

Rereadiing Belleau

\section{Lo teórico, la política, Bajtín y la sociocrítica}

\section{Leer de nuevo a Belleau}

\section{Pierre Popovic}

Volume 42, numéro 1 (124), automne 2016

André Belleau I : relire l'essayiste

URI : https://id.erudit.org/iderudit/1038589ar

DOI : https://doi.org/10.7202/1038589ar

Aller au sommaire du numéro

\section{Éditeur(s)}

Université du Québec à Montréal

\section{ISSN}

0318-9201 (imprimé)

1705-933X (numérique)

Découvrir la revue

\section{Citer cet article}

Popovic, P. (2016). Le théorique, la politique, Bakhtine et la sociocritique : relire Belleau. Voix et Images, 42(1), 77-85. https://doi.org/10.7202/1038589ar
Résumé de l'article

Précoce introducteur des travaux de Mikhaïl Bakhtine dans le monde occidental des études de lettres, André Belleau a entretenu avec la théorie littéraire une relation curieuse, gourmande, passionnée, toujours orientée vers ce qui l'intéressait le plus : transformer le texte de littérature en question de manière à le rendre susceptible d'éclairer un peu le monde tel qu'il est connu au moment où il y fait son apparition. Mais ce rapport au théorique est aussi un rapport complexe, où entrent parfois de l'insatisfaction, des désirs irréalisés de dépassement, du malaise. L'étude en cherche l'origine et la trouve à la fois du côté politique et du côté de l'émergence d'une problématique que le destin ne lui a pas permis de développer, celle des relations entre la culture populaire et la culture savante. Nul doute qu'elle l'aurait conduit à élaborer lui-même des concepts propres à son traitement. 


\section{LE THÉORIQUE，LA POLITIQUE，BAKHTINE \\ ET LA SOCIOCRITIQUE}

Relire Belleau

$+++$

\section{PIERRE POPOVIC}

Université de Montréal/Centre de recherche interuniversitaire en sociocritique des textes (CRIST)

Si la réputation de théoricien qui accompagne André Belleau n'est pas usurpée, un regard rétrospectif sur ses textes indique que son rapport avec la théorie était complexe, parfois difficile, quelquefois malaisé. Cela se remarque à quatre traits ou indices particuliers. Le premier est qu'il n'a jamais proposé d'élaboration conceptuelle qui lui soit propre. Il a repris des concepts et les a étudiés en même temps qu'il les utilisait, mais il n'en a pas élaboré lui-même un, comme Duchet ou Angenot l'ont fait par exemple pour le sociogramme ou le discours social. Le second trait est que ses essais comportent des assertions parfois étranges. À l'exemple de celle-ci:

Je défie tout simplement quiconque de me décrire en quelques pages la MÉTHODE d'Auerbach dans Mimésis, de Lukàcs dans La théorie du roman (sa méthode, pas ses fondements philosophiques!), d'Adorno dans les Noten zur Literatur, de Bakhtine dans son Rabelais ${ }^{1}$.

Il n'y a aucune difficulté ni d'ailleurs aucun mérite à relever un tel défi à condition de tenir compte du fait que les fondements philosophiques de la pensée de ces trois auteurs comportaient et impliquaient des choix méthodologiques. Pour ne prendre qu'un exemple, La théorie du roman du «jeune» Lukàcs articule des éléments tirés de la dialectique hégélienne, de la méthode historique, de l'épistémologie wébérienne et de la philologie allemande ${ }^{2}$, éléments qui sont hiérarchisés dans cet ordre ${ }^{3}$. Cette réserve sur l'identification des méthodes doit sans doute quelque chose aux essayistes qui entourent Belleau à la revue Liberté, qui ne cessent en ces années-là (fin des années 1970 et années 1980) de fustiger et de moquer les théories littéraires de quelque obédience qu'elles soient sans jamais les discuter vraiment. Sous

1 André Belleau, «La sociocritique et la littérature québécoise», Y a-t-il un intellectuel dans la salle?, Montréal, Primeur, coll. «L'échiquier», 1984, p. 158. Désormais, les références à cet ouvrage seront indiquées par le sigle $Y$ suivi du folio, et placées entre parenthèses dans le texte.

2 À laquelle Karl Marx avait également été formé. Cette philologie allemande ne se limite pas à l'établissement critique des textes, elle s'intéresse aussi aux formes littéraires, ainsi qu'à la sémantique et à l'interprétation des œuvres.

3 La matrice principale est la dialectique de l'idéalisme et du matérialisme. 
les plumes de François Ricard, de Jean Larose et de quelques autres, ces approches théoriques sont globalement ramenées à des jargons, des trissotinades et des préciosités qui relèvent du pétage de bretelles et nient les splendeurs humanistes de l'héritage des lettrés bien dotés ${ }^{4}$. Le troisième indice de cet inconfort théorique est la présence de contradictions non levées. Dire à un moment donné de Barthes qu'il fait aussi œuvre de sociologue et, à un autre moment, qu'il néglige tout rapport des œuvres avec la société; emprunter la notion d'isotopie à Greimas, mais la garder en surface de langue sans tenir compte du fait qu'elle désigne un réseau de sens infralinguistique ${ }^{5}$; souhaiter à la sociocritique qu' elle fasse de la sémiotique du texte, mais rejeter ailleurs la sémiotique parce qu'elle ignore les rapports du texte avec la régulation des langages sociaux chère à Bakhtine ${ }^{6}$; de telles contradictions sont parfaitement tolérables dans des essais littéraires, mais elles s'avèrent plus problématiques dans des essais animés par une intention théorique. Enfin, le quatrième trait consiste en une tendance œcuménique, redondante et gourmande. Belleau inventorie et commente tant de travaux complexes que son lecteur est à la fois épaté par sa capacité de synthèse et sceptique devant la cohérence générale de la cohabitation des auteurs convoqués. Cela n'a pas échappé à Gilles Marcotte, qui, dans un texte d'hommage de 1987, se rappelle avec une tendresse malicieuse que, pour deux pages consacrées à Roger Lemelin - qui, dit-il, «en a perdu son latin s'il a lu ce passage» -, sont assignés à comparution beaucoup de gens:

\begin{abstract}
D'abord un petit détour par la peinture, Van Eyck et Velasquez. Puis: Gérard Genette parlant de Proust, Michel Zéraffa de Hermann Broch, l'Ulysse de Joyce, Jean Ricardou, Françoise Van Rossum-Guyon parlant de La modification de Michel Butor, et Hermann Hesse, et Thomas Mann, pour finir par un dernier théoricien, Claude Bremond. Et André Belleau de conclure: «Denis Boucher appartient un peu à cette famille ${ }^{7} \ldots »$
\end{abstract}

En somme, André Belleau est un théoricien belge. Il y a en effet dans la largeur de ce regroupement une pathologie qui, pour un compatriote de Maurane et de Stromae, est très reconnaissable. Elle est celle qui consiste à toujours craindre qu'il n'y en ait pas assez, à toujours craindre de rater quelque chose ou quelqu'un. Il en résulte immanquablement des effets consensuels bizarres ${ }^{8}$.

4 Qu'il y ait eu des abus de jargon, de trissotinerie et de préciosité, ce n'est pas faux. Le problème vient du caractère global et complaisant de l'accusation.

5 C'est-à-dire: qui se diffuse au niveau des sèmes.

6 Lequel parlait de régulation réciproque dans tout acte de communication. Voir Mikhail Bakhtine et V[alentin] N[ikolaievitch] Volochinov, Le marxisme et la philosophie du langage. Essai d'application de la méthode sociologique en linguistique, traduit du russe par Marina Yaguello, Paris, Éditions de Minuit, coll. «Sens commun», 1977, $232 \mathrm{p}$.

7 Gilles Marcotte, «Pour mémoire», Liberté, vol. XXIX, n 1, février 1987, p. 42.

8 L'un des beaux exemples belges de cela est L'institution de la littérature de Jacques Dubois (Paris/Bruxelles, Fernand Nathan/Éditions Labor, coll. «Dossier média», 1978, 188 p.). L'introduction de cet ouvrage passe en revue divers critiques qui ont parlé d'institution ou, pour le dernier qui sera nommé, de quelque chose d'équivalent. Se succèdent Roland Barthes, Theodor Adorno, Jean-Paul Sartre, l'institutionnaliste René Lourau et Pierre Bourdieu. Après les avoir commentés, Dubois conclut qu'il y a entre les uns et les autres 
Les traits et indices qui viennent d'être relevés ne l'ont pas été pour pointer quelque manquement ou défaillance dans le maniement des outils théoriques chez Belleau, loin de là. Ils sont en fait le lot de tous les théoriciens qui ne se bornent pas à être de simples collectionneurs. Les travaux d'épistémologie de Max Weber donnent la raison de telles difficultés: en sciences humaines, les concepts ne recouvrent jamais pleinement les phénomènes qu'ils désignent. En d'autres termes, ils ont toujours des problèmes d'orthogonalité, de discrétion et d'extensibilité, ce que n'ont pas leurs équivalents des sciences naturelles. De tels problèmes arrivent de surcroît particulièrement quand le théoricien est condamné ou se condamne à écrire des textes courts, ce qui est le cas de Belleau. En dire et réunir trop sont peut-être les traits d'une pathologie de la périphérie francophone (ou autre), mais en l'occurrence ce sont aussi les conséquences du choix du genre de l'essai et du format serré des textes publiés dans Liberté. Pour aller vers plus de précision, pour engager soi-même un débat avec des critiques choisis, il faut avoir plus de millage à faire, prendre plus de temps et plus de distance, être moins pédagogique, quitter la délibération de l'essai pour l'argumentation critique et l'enchaînement serré des inférences théoriques. Il suffit de lire successivement deux pages de Belleau et deux pages de Marc Angenot pour mesurer la différence.

Mais si elles ont ainsi des causes épistémiques et matérielles, ces difficultés disent quelque chose de beaucoup plus important sur le rapport entretenu par André Belleau avec la théorie. Les traces les plus claires s'en trouvent dans son utilisation des travaux de Mikhail Bakhtine et dans ses premiers essais pour appliquer la théorie du critique russe à la littérature québécoise. Soit l'article fameux sur «La dimension carnavalesque du roman québécois» ( $Y, 166-174)$. Ce texte résume les grandes lignes du Rabelais de Bakhtine et pose que «la carnavalisation désigne la transposition dans la littérature de la culture populaire conçue comme vision complète du monde et non seulement la survivance textuelle de résidus carnavalesques» (Y,167). Dans cet esprit, l'affirmation d'une présence importante de carnavalesque dans le roman québécois pourra être soutenue sous réserve d'y rencontrer « des phénomènes de carnavalisation: dispositifs narratologiques embrouillés, interactions dialogiques complexes, réseaux sémantiques dessinant le "corps grotesque" ( $Y, 167)$. Ramené à cinq éléments impératifs, le diagnostic de carnavalesque implique une "exigence vitale et universelle de participation», «la suppression joyeuse des distances entre

quelque convergence menant vers l'idée que la littérature est une institution. Dès la parution de cet essai, cette convocation d'autorités était plus que suspecte. Le mot «institution» figurait bien chez Barthes, Adorno, Sartre et Lourau, mais il ne désignait pas la même chose et ne relevait pas des mêmes batteries d'hypothèses dans les quatre cas. Quant au cinquième nommé, Bourdieu, qui connaissait sans aucun doute la critique incendiaire et définitive du concept d'origine durkheimienne d'«institution» faite par Georges Gurvitch, il n'allait pas voir d'un très bon œil la possibilité que «l'institution de la littérature» destitue son concept de «champ littéraire». Il ne lui consacrera d'ailleurs qu'une note assassine, qui commence clairement

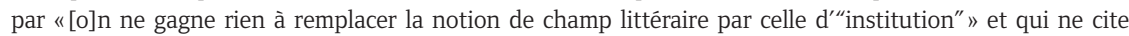
pas le livre de Dubois (Pierre Bourdieu, Les règles de l'art. Genèse et structure du champ littéraire, Paris, Éditions du Seuil, 1992, p. 321, note 21). C'est François Provenzano qui, dans Vies et mort de la francophonie. Une politique française de la langue et de la littérature (Bruxelles, Impressions nouvelles, coll. «Réflexions faites », 2011, 281 p.), a le mieux saisi ce phénomène d'œcuménisme en observant la différence - de nature idéologique - entre le centre et la périphérie: ce qui à Paris fait débat et crée du dissensus se rabote et devient consensuel à Liège ou à Montréal. Le mot "sociocritique» lui-même a été par le passé l'objet d'un tel brouillage, et il a fallu quelques efforts — d'ailleurs à recommencer périodiquement — pour le tirer de là. 
les hommes», «l'expression concrète des sentiments refoulés», «le rapprochement de ce que la vie quotidienne séparait» et une pratique abondante de «l'inconvenance parodique et profanatrice» (Y,167-169). Bien. Cependant, dès que le regard s'intéresse d'un peu trop près aux exemples étudiés, les choses commencent à grincer, ainsi que le montrent les deux cas qui suivent.

«L'expression concrète des sentiments refoulés» est donnée pour un écart pris avec la convention réaliste. Cette dernière est de mise dans les propos de la Mère Plouffe, lesquels sont attendus et par suite vraisemblables chez Roger Lemelin. Ce n'est pas le cas dans le discours de Mireille, la secrétaire dans D'Amour, P.Q. de Jacques Godbout, lequel discours n'a rien de commun avec «notre expérience ou connaissance du rapport patron-employée dans la vie réelle» $(Y, 168)$. Cette «relation secrétaire-patron normalement commandée par la vraisemblance romanesque est remplacée par la relation [entre le] "langage dans le roman"» [et un] "langage social externe" dans une joyeuse relativisation carnavalesque", ce qui ne l'empêche pas de devenir chez Mireille «une sorte de re-création esthétique du langage et de l'idéologie de toute une partie de la jeunesse québécoise» $(Y, 168)$. L'analyse est sans doute défendable, mais où est passée la culture populaire dans cette affaire? La relation patron-employée est tout de même fort spéciale. Thomas d'Amour, le patron, est écrivain. Mireille est non seulement sa secrétaire ${ }^{9}$, mais aussi sa lectrice, elle lit ses manuscrits et donne son avis, qu'elle partage avec franchise: «Votre manuscrit, à mon avis, c'est dans le genre chiant décadent, poésie de bord de mer, comme ce que tout le monde écrit en vacances à Percé, ou au retour d'un voyage dans les Uropes ${ }^{10}$.» Il y a là une liberté de ton, un texte en débat avec son temps, mais un renversement carnavalesque, non. Il est aussi loisible de se demander quel rapport cette intégration d'un discours idéologique - qui n'apparaît pas seulement lié à une "partie de la jeunesse québécoise» en conjoncture, à moins d'étendre la notion de jeunesse jusqu'à quarante ou cinquante ans ${ }^{11}$ - entretient avec telle chose que «l'expression concrète des sentiments refoulés». N'est-il pas également soutenable que le discours de Mireille est forcément vraisemblable s'il traduit une idéologie répandue dans une partie de la jeunesse québécoise? Une étude, très vertueuse, de Brigitte Seyfrid, directement inspirée de Belleau, affirme que D'Amour, P.Q. est un roman animé par «une force positive et vitale», qui comporte «un aspect régénérateur et libérateur, inhérent à la conception bakhtinienne du carnavalesque ${ }^{12}$ ». À ce compte, Le sang

9 Au sens où il y a eu et où il y a encore des secrétaires d'écrivain, fonction bien à part d'un secrétariat d'entreprise ou d'institution.

10 Jacques Godbout, D’Amour, P.Q., Montréal/Paris, Hurtubise HMH/Éditions du Seuil, 1972, 156 p.

11 L'ídée d'un changement social passant par une libération du langage est typique de la Révolution tranquille, ainsi que le signalent un slogan comme "on est six millions, faut se parler», le matériel publicitaire des éditions de l'Hexagone (où il s'agit d'exprimer ce qu'il y a «dans le cœur de nos vies») naguère étudié par Gilles Marcotte (voir «L'Hexagone et compagnie», Littérature et circonstances, Montréal, l'Hexagone, coll. «Essais littéraires», 1989, p. 113-122), l'idéologème «dire, c'est faire» ou l'emballement pour la communication transitive entre individus qui anime la société du temps (voir sur ces sujets Micheline Cambron, Une société, un récit. Discours culturel au Québec, 1967-1976, Montréal, l'Hexagone, coll. «Essais littéraires», 1989, 201 p.).

12 Brigitte Seyfrid, «Polyphonie, plurilinguisme et vision carnavalesque dans $D^{\prime}$ Amour, P.Q. de Jacques Godbout», Voix et Images, vol. XXI, n 3, printemps 1996, p. 558. 
des autres de Simone de Beauvoir, qui aurait été bien surprise de l'apprendre, est un texte bakhtinien. Car cette lecture laisse diantrement sceptique. D'une part, le roman de Godbout est ironique de bout en bout, et souvent lourdement; d'autre part, et ceci touche tous les autres exemples de Belleau, il n'y a pas ici ce rire de fête, emporté et systémique, il n'y a pas non plus ces corps productifs et hyperactifs dont toutes les ouvertures communiquent avec le monde. À l'inverse de ce qui se passe chez Rabelais, les organes jouissifs sont linguistiquement frileux et la générosité des humeurs organiques est chiche chez l'auteur de La concierge du Panthéon (2006).

La «suppression joyeuse des distances entre les hommes» rencontrerait un «magnifique exemple» dans L'amélanchier de Jacques Ferron, «dont le narrateur présente ainsi un personnage: "Fin causeur et fils de cultivateur, il se nommait Léon. Léon de Portanqueu, esquire." " $(Y, 168)$ Analysant cette déclinaison d'identité, Belleau voit en elle une "“construction hybride complexe"» (Bakhtine) qui «rapproche si bien les classes qu' elle les attribue toutes à une seule personne: les intellectuels, les paysans, la noblesse française, la gentry anglaise » $(Y, 168)$. Paradoxalement, le genre qui embrasse le mieux cette conception n'est pas le roman carnavalesque, mais plutôt le conte fantaisiste, au sens noble et esthétique du mot «fantaisiste» ou «fantaisie $»^{13}$. Mais il y a surtout que la construction poly-indicielle n'attribue pas toutes les classes sociales au personnage et elle ne les rapproche pas le moins du monde dans une allégorie joyeuse. Elle indique plutôt une confusion inconsciente, un brouillage des valeurs dont le père est le symptôme et la société environnante le fleuron. Ledit Léon est un père qui n'a rien de Grandgousier, lui dont sa fille Tinamer dit qu'il est « une brute qui méritait bien son nom de pape ${ }^{14}{ }$. Belleau termine l'étude de cet exemple par cette assertion: «Nous apprenons plus tard que Léon de Portanqueu est pompier dans une banlieue de Montréal.» Ah! S'il était pompier, ça tomberait bien, car il serait lié au feu, et il est de notoriété critique depuis Platon que la maîtrise du feu est intimement liée à la création artistique, et il n'est pas moins notoire depuis Rabelais, qui avait lu Platon, et depuis Bakhtine, qui a lu Rabelais qui a lu Platon, que les aventures des amis pantagruéliques de tout honnête homme cultivent une grande représentation mythologique, celle de l'incendie régénérateur, du feu comme symbole de la régénération du monde. Véritable fête pour un pompier. Le problème, c'est que ce n'est pas vrai. Léon de Portanqueu n'est pas pompier dans L'amélanchier, mais geôlier, ce qui n'est pas vraiment la même chose. Léon se fait à un moment donné traiter de pompier, et c'est un autre symbole du feu qui le lui dit, sa volcanique femme Etna. Tinamer rapporte en effet cette conversation de ses parents:

[Quand Léon de Portanqueu] disait à ma mère: «Femme de peu de foi, indigne du héros que toi-même tu déshabillais autrefois, qu'as-tu fait de ta main avisée et de tes flammes juvéniles?»

Etna lui répondait:

13 Cette catégorie s'est apparemment perdue dans la littérature française, qui eut pourtant ses «poètes fantaisistes», alors qu'elle s'est maintenue dans d'autres littératures, hispanophones, par exemple, et pourrait peut-être servir pour la littérature québécoise, avec Ferron comme grand illustrateur. Jacques Ferron, L'amélanchier, Montréal, Éditions du Jour, 1970, p. 25. 
- Léon de Portanqueu, demande-le au pompier que j'ai épousé. Il m'a si bien éteinte que c'est miracle que ma cuisinière électrique fonctionne encore ${ }^{15}$.

L'ennui avec le pompier réside dans le fait de son rapport au feu. Il éteint, mais n'allume guère. En l'occurrence, l'érotisation du dialogue propage non pas des flammes, mais bien des cendres sur l'entièreté du récit. Celui-ci est drôle et substantifique, rien à dire là-dessus, mais son rire n'est pas un rire carnavalesque. À cela s'ajoute que le corps dans les récits de Ferron n'a rien du corps rabelaisien, communiquant de toutes ses forces avec le monde, où tous les trous, des yeux et des oreilles au boyau cullier, sont ouverts en sorte que le corps est fécondé par le dehors et le dehors fertilisé par les cris, les regards, les déjections et les sécrétions corporelles. Quand il rentre du travail, Léon de Portanqueu se débarrasse de ses vêtements et se met debout devant la fenêtre. Dans la même situation, Gargantua verrait les Pyrénées à 800 km de distance. Léon de Portanqueu, lui, ne voit rien: «Un fourré de cèdres, d'aubépines et de cornouillers s'était développé devant la maison, qui, sans la rendre plus accueillante qu'il ne fallait, lui masquait à peu près la rue, ses charriots patrouilleurs et ses vilains passants ${ }^{16}$.» Le carnavalesque est décidément bien loin, et le pompier n'a rien d'un pyromane ou d'un artificier.

Il faut prendre ces scories interprétatives au sérieux, car elles indiquent un élément remarquable de l'écriture de Belleau, présent aussi bien dans ce qu'elle a de plus lumineux que dans les passages où dérivent du raté ou de la contradiction. Il y a que cette écriture cherche quelque chose qu'elle ne trouve pas. Elle a beau lire et lire et lire des tas de gens et des tas de travaux, elle est animée par une question qui la taraude, que ni Barthes ni Goldmann ni aucun autre auteur du vaste corpus critique inventorié ne (se) pose. Cette question est profondément politique, et c'est à cause de ce caractère politique qu'elle est unique et que Belleau a tant besoin de Bakhtine. À l'épreuve des faits, nourrie par les travaux de Bakhtine et Marcotte, la quête théorique de Belleau le mène directement à la sociocritique. Elle est vectorialisée par un désir politique que deux textes publiés juste avant et juste après le référendum de mai 1980 donnent à lire, "L'esthétique du "oui" » $(Y, 78-79)$ et «On ne meurt pas de mourir» $(Y, 80-81)$, ce dernier étant peut-être l'essai le plus émouvant qu'il ait écrit.

«L'esthétique du "oui" » procède à une construction de l'ennemi qui, à défaut d'une théorie sociopolitique qui justifierait l'indépendance, devient la raison fondamentale de son choix. Cet ennemi se compose de quatre éléments : des gens précis, avec lesquels paraître ou fricoter serait une trahison de soi (le cardinal Carter, Marc Lalonde, Roger Lemelin, Charles Bronfman, Claude Ryan, Marc Carrière et Paul Desmarais ${ }^{17}$ ); le Conseil du patronat; la Chambre de commerce; les grands présidents, les grands intérêts, les multinationales. Ces gens, ces associations, ces entreprises sont associés à la laideur de l'existence et, pour la plupart, au pouvoir de l'argent. Face à cette hideur se dresse le «oui» pour l'indépendance, laquelle se justifie esthétiquement,

15 Ibid., p. 33.

16 Ibid., p. 32.

17 Avec son côté «liste» qui n'est guère réjouissant, cette séquence n'a aucune valeur argumentative réelle. Un intellectuel fédéraliste comme Gilles Marcotte n'aurait bien entendu eu aucune peine à dresser une liste équivalente de partisans de l'indépendance avec lesquels il aurait refusé de dîner. 
par sa beauté vitale en quelque sorte, Belleau parlant d'une esthétique du «oui» qui équivaudrait à une esthétique "élémentaire de l'existence» ( $Y$, 78). Il est à noter que cet autre, cet ennemi intérieur, ce n'est pas «la droite». Ce n'est pas comme cela que la prose en parle. L'essayiste souligne qu'il y a dans le camp du «oui» une hétérogénéité idéologique et laisse entendre son désaccord avec certaines de ses composantes (par exemple celle d' «un québécocentrisme réactionnaire tout à fait dans la tradition nationaliste» $[Y, 78])$. Il se dit en effet antinationaliste et fédéraliste ${ }^{18}$, et se trouve ainsi en porte-à-faux avec les courants nationalistes du Parti québécois. Il passe par-dessus ce différend en accordant à ce parti un rôle historique très au-dessus des attentes de la petite bourgeoisie qui constitue sa base électorale. Cette argumentation est d'évidence fragile, et Belleau en est manifestement conscient. Que les partisans de l'indépendance soient, ne serait-ce que majoritairement, contre la Chambre de commerce et le Conseil du patronat appellerait une vérification que le devenir ultérieur du Parti québécois rend improbable. C'est pourquoi il justifie le recours à la raison esthétique par deux affirmations étonnantes et légèrement contradictoires: l'indépendance "offre [une résistance] à la pensée théorique» et «nous n'avons pas encore une théorie valable de l'indépendance nationale ${ }^{19}$ " $(Y, 79)$. Fort de quoi, il conclut que " [l]'indépendance, tout comme la liberté, n'est pas une question théorique mais pratique, disons existentielle» $(Y, 79)$, ce qui ferme la boucle et monte en épingle un argument définitif qui peut se résumer comme suit: l'indépendance, c'est beau. Mais ce qui en profondeur force l'attention dans ce texte, ce n'est pas l'indépendance, ni la question de savoir si Paul Desmarais est, du point de vue d'une "esthétique élémentaire de l'existence», plus ou moins supportable que Pierre-Karl Péladeau ou que son père; $c^{\prime}$ est le fait qu'il établit une corrélation forte entre l'esthétique, le politique et la théorie.

Il en va de même dans "On ne meurt pas de mourir». Il y a beaucoup de tristesse et une amertume courageusement contenue dans ce texte, lequel arbore quatre temps forts. Premier temps: face à la défaite référendaire, le texte insiste sur la nécessité de refuser d'accuser le peuple de quoi que ce soit. L'essayiste prouve ici, s'il en était besoin, qu'il est un penseur démocrate et s'éloigne radicalement, par rétroaction, des inévitables discours qui imputeront la victoire du «non» à des réflexes ataviques, à des manœuvres frauduleuses de l'adversaire, à une peur pathologique héritée d'un passé colonial ou au règne en longue durée d'un catholicisme moralement répressif.

Vient ensuite une explication du sens de la victoire du «non»: c'est à ses yeux la victoire du même sur l'autre, en un nivellement des différences typique de la conformité nord-américaine. À nouveau survient ici une phrase étonnante, où le syntagme de la «théorie valable» fait retour. Tout se passe comme s'il y avait quelque chose d'impensable dans la victoire et dans la défaite: «nous n'avons pas une théorie valable de la causalité historique ${ }^{20} »(Y, 80)$.

18 Ce mot, destiné à priver l'adversaire du label monopolisé de son identité, renvoie à l'idée selon laquelle un Québec souverain partagerait «diverses compétences avec les collectivités régionales» $(Y, 78)$.

19 Ce qui supprime curieusement les assises «théoriques» que le mouvement indépendantiste cherchait en conjoncture dans les écrits des penseurs anticolonialistes (Memmi, Fanon, Césaire, etc.).

20 En synchronie, Cornelius Castoriadis aurait pu lui fournir une explication à cette absence (qui dépasse de loin le cas du Québec). Ses travaux sur l'imaginaire social reposent en effet sur le constat que l'évolution 
$\mathrm{Au}$ troisième temps, le texte énumère les conséquences culturelles de la défaite du "oui». 1. Le Québécois reste et demeure "non identifiable» («Je ne sais pas qui je suis.») $(Y, 80)$. 2. Ce qui avait servi de «moteur utopique» $(Y, 81)$ durant vingt ans vient de s'arrêter brutalement. C'est dans ce passage que la question esthétique fait retour, mais elle concerne cette fois directement la littérature et la façon de la lire. Après avoir écrit que l'utopie, quoique «[i]ndispensable à la vie, [...] ne la modifie pas», Belleau ajoute que "la littérature est précisément ce qui n’arrive jamais», en sorte que "[t]oute une relecture s'impose des poètes depuis la fin des années cinquante» $(Y, 81)$. 3. Il est devenu vain et menteur de mettre de l'avant «les aspects collectifs de [la] culture [québécoise]» $(Y, 81)$, sous peine de transformer cette culture en folklore.

Le quatrième temps est celui du retour sur soi. Belleau ne se reconnaît plus qu'à et qu'en deux choses: une identité montréalaise et une appartenance à la bourgeoisie, avant de conclure en conjuguant le conditionnel passé première forme et un futur d'outre-tombe: «J'aurais voulu que nous soyons Québécois. Cela n'arrivera pas de mon vivant.»

Le mariage à trois, de l'esthétique, de la politique et du théorique, est aussi présent dans "On ne meurt pas de mourir». Mais alors qu'il débouchait sur une pratique et des choix vitaux dans «L'esthétique du "oui" », il s'envase cette fois dans une résignation douloureuse. Relire aujourd'hui ce texte d'un lendemain de défaite donne l'impression de découvrir non seulement un diagnostic de suicide collectif (marqué par l'indéfini «on» du titre), mais aussi l'annonce d'un suicide personnel, à la fois intellectuel et existentiel. Car il y a deux contre-phrases possibles à l'intitulé du texte. L'une dira "On ne meurt pas de mourir. On meurt de vivre»; l'autre dira «On ne meurt pas de mourir. On meurt de se tuer». Belleau ne s'est pas tué. Sur le plan intellectuel, ce sont ses élans vers Bakhtine et vers la sociocritique qui le sauvent.

Il faut revenir pour le comprendre sur ces phrases: «nous n'avons pas une théorie valable de la causalité historique»; «nous n'avons pas encore une théorie valable de l'indépendance nationale». Mais, seigneur, de quoi «avonsnous» donc une "théorie valable»? Ou, mieux, à défaut de théorie valable, $\mathrm{qu}^{\prime}$ «avons-nous»?

«Nous» - qui est ce «nous»? - avons ceci:

On aura remarqué que Gilles Marcotte ne sort pas de l'ordre du discours. Comment une sociocritique pourrait-elle y parvenir? Le hors-texte est lui-même texte. [...] Le roman à l'imparfait de Gilles Marcotte est le premier exemple d'une sociocritique de la littérature d'ici qui envisage résolument la société elle-même comme un texte ${ }^{21}$.

historique n'est pas une suite de causes et d'effets prévisibles, puisque les mêmes causes ne produisent manifestement pas les mêmes effets selon les sociétés et selon les époques. Voir Cornelius Castoriadis, L'institution imaginaire de la société, Paris, Éditions du Seuil, 1975, 538 p.

21 André Belleau, «La sociocritique et la littérature québécoise» $(Y, 163)$. Il dira ailleurs que cet essai de Marcotte annonçait la défaite du «oui» en mai 1980. 
Belleau n'a jamais été aussi clair sur la spécificité épistémologique de la sociocritique, et la lucidité de cette phrase, en plus de nombre de ses travaux, fait de lui l'un des quatre piliers de «L'École de Montréal ${ }^{22}$ ».

Et «nous» avons Bakhtine. Au fur et à mesure que Belleau avance, l'idée que l'une des altérités majeures des textes d'ici est la culture populaire québécoise s'affirme et se précise. C'est elle qu'il va falloir penser. Bakhtine, sans nul doute, est la première pierre angulaire de ce travail, et son Rabelais, à coup sûr, est irremplaçable. Mais il est désormais (1980) clair qu'il faudra élaborer des outils notionnels et conceptuels propres qui permettront de penser cette culture populaire d'ici. Cette dernière est triplement singulière: elle l'est fondamentalement en raison de son histoire et de l'évolution de la semiosis sociale ${ }^{23}$ au (Canada français puis au) Québec; elle l'est par ses relations originales avec la culture savante; elle l'est parce qu'elle est dès toujours terreautée par les apports d'alluvions, de colluvions et de sédiments issus des premiers habitants de ce coin d'Amérique et des innombrables immigrants successifs. Dans l'esprit de l'auteur de Surprendre les voix, titre qui correspond si bien à la sociocritique, ce recours à la multiculture populaire québécoise est une exigence spirituelle et sociopolitique. C'est une façon de sauver le peuple québécois du suicide collectif, une façon d'éviter l'accusation d'irresponsabilité historique, une façon d'écarter le risque de devenir non identifiable et invisible, c'est là qu'est toute l'affaire. Si Belleau avait eu la chance de vivre plus longtemps, il aurait travaillé sur ce que c'est vraiment que cette culture composite en longue durée et aurait à coup sûr été très sensible aux rencontres qui sont organisées depuis quatre ans entre ethnocriticiens et sociocriticiens à Montréal. Léon de Portanqueu n'était pas pompier, il y avait erreur sur le personnage; mais l'erreur n'était pas qu'une banale inattention. Car le texte sur «La dimension carnavalesque du roman québécois» est de 1983. Le mot "pompier» peut dès lors être tenu pour un acte manqué. Pour Belleau, le référendum de 1980 a éteint le lumignon de l'utopie. Aller étudier la relation intersémiotique entre culture populaire et texte littéraire, c'était une manière de rallumer une flamme et, qui sait, de mettre en chantier l'élaboration d'une «théorie valable», qui allait être animée par des notions et des concepts nécessairement faits maison, une théorie enfin signée "André Belleau ».

22 Voir Pierre Popovic, «La sociocritique: présupposés, visées, cadre heuristique - L'École de Montréal», Revue des sciences humaines, $\mathrm{n}^{\circ} 299$, juillet-septembre 2010, p. 13-29.

23 Concept-clé de la sociocritique, la semiosis sociale désigne les multiples façons par lesquelles une société se représente ce qu'elle est, ce qu'elle a été et ce qu'elle pourrait devenir au moyen de tous les langages dont elle dispose. Pour les modalités de son utilisation, voir par exemple Pierre Popovic, La mélancolie des Misérables. Essai de sociocritique, Montréal, Le Quartanier, coll. «Erres essais», 2013, 310 p. 